\title{
Diffusion Tensor Imaging in Anxiety Disorders
}

\author{
Elliot Ayling • Moji Aghajani • Jean-Paul Fouche • \\ Nic van der Wee
}

Published online: 30 March 2012

(C) Springer Science+Business Media, LLC 2012

\begin{abstract}
Diffusion tensor imaging (DTI) can be used to examine the structural integrity of regional white matter and to map white matter tracts. DTI studies have been performed in several psychiatric disorders, especially in those for which a developmental or a neuropsychiatric component was postulated. Thus far, the use of DTI has been very limited in panic disorder, social anxiety disorder, and generalized anxiety disorder, and somewhat more extensive in post-traumatic stress disorder and obsessive-compulsive
\end{abstract}

\section{E. Ayling}

Leiden University Medical Center,

Albinusdreef 2,

2333 ZA Leiden, The Netherlands

e-mail: staziac@hotmail.fr

\section{Aghajani}

Department of Child and Adolescent Psychiatry,

Curium-Leiden University Medical Center,

Endegeesterstraatweg 27,

2342 AK Oegstgeest, The Netherlands

e-mail:m.aghajani@curium.nl

\section{J.-P. Fouche}

Department of Human Biology and Department of Psychiatry,

University of Cape Town,

Anzio Road, Observatory,

7925 Cape Town, South Africa

e-mail: jfouche@sun.ac.za

\section{J.-P. Fouche}

Department of Psychiatry, Stellenbosch University,

Francie van Zijl, Tygerberg,

7500 Cape Town, South Africa

\section{N. van der Wee $(\bowtie)$}

Department of Psychiatry and Leiden Institute

for Brain and Cognition, Leiden University Medical Center,

Albinusdreef 2,

2333 ZA Leiden, The Netherlands

e-mail: n.j.a.van_der_wee@lumc.nl disorder. In most anxiety disorders, the results of DTI studies are in line with other structural and functional MRI findings and can be interpreted within the frameworks of existing models for the neurocircuitry of the various disorders. DTI findings could further enrich neurobiological models for anxiety disorders, although replication is often warranted, and studies in pediatric populations are lagging behind remarkably.

Keywords Diffusion tensor imaging $\cdot$ DTI $\cdot$ Anxiety disorders · Neurocircuitry · Panic disorder · Social anxiety disorder - Generalized anxiety disorder - Post-traumatic stress disorder - Obsessive-compulsive disorder - Internalizing disorders $\cdot$ Trait anxiety

\section{Introduction}

The first studies in which diffusion MRI was used were published in the mid-1980s. Diffusion MRI can be used to examine several aspects of the diffusion of molecules, also called Brownian motion, in biological tissues. Without obstacles, the diffusion of molecules would be free and isotropic, but in biological tissues, there is an interaction of the molecules with membranes and other macromolecules. Changes in the diffusion pattern of molecules convey information on the surrounding microstructure. Usually the diffusion of water molecules is studied, but this can be applied to other metabolites as well. Clinical applications of diffusion MRI have become important in the early detection and treatment of brain ischemia.

Diffusion tensor imaging (DTI) is based on the principle that the diffusion of water molecules is not random (or anisotropic) in tissues with an internal fibrous structure, such as the axons in the white matter of the brain. Water 
molecules will diffuse more easily along the direction of the axons, and more slowly perpendicular to the direction of the axons. By measuring the diffusion of water molecules in a voxel in the brain from several directions, usually six or more, and using vector algorithms, a diffusion tensor can be calculated. The tensor conveys information on the degree of local anisotropy (the fractional anisotropy [FA]) and the principal direction of local diffusion. Between-group differences in FA in a certain region of the brain reflect differences in the structural organization or integrity of that region. Higher FA values are thought to reflect a higher degree of neuronal organization There are other measures of diffusivity, but FA is a commonly used one.

By examining neighboring voxels, the diffusion tensor can also be used for tractography (ie, mapping the trajectory of axon bundles through the brain). For tractography, the diffusion is measured in a large number of directions, and hence a longer acquisition time is usually needed.

DTI studies have been performed in several psychiatric disorders, especially in those for which a developmental or a neuropsychiatric component has been postulated, such as schizophrenia. In this paper, we review DTI studies in the $D S M-I V$ anxiety disorders.

\section{Diffusion Tensor Imaging in Anxiety Disorders}

\section{Panic Disorder}

A panic attack is a discrete episode of intense fear, discomfort, and sympathetic nervous system arousal that occurs in the absence of true danger. Patients with panic disorder (PD) experience recurrent, unexpected panic attacks along with a persistent concern about having future attacks. They also worry about the implications of the attacks or show a significant change in behavior related to the attacks [1].

The "fear network," involving the amygdala, hippocampus, thalamus, insula, brain stem structures, and several medial, and prefrontal areas is the key brain circuit involved in PD. The frontal cortex is thought to have a regulatory role in this fear network by "dampening" activity of the amygdala, and is also involved in fear conditioning [2]. More specifically, areas within the anterior cingulate cortex (ACC) appear to be involved in the neurocircuitry of PD. Studies using functional MRI have shown hyperresponsiveness in both rostral and dorsal divisions of the ACC in PD. The very limited number of structural volumetric studies in PD have shown reductions in volume of both the rostral and dorsal ACC [3-5].

We could only identify one study that has used DTI to study cerebral abnormalities in PD. Han et al. [6] in 2008 used a region of interest (ROI) approach to assess structural white matter integrity in anterior and posterior cingulate regions in 24 adult patients with PD without comorbidity and 24 matched controls. All patients used medication. The authors found a significant increase in the FA values of the left anterior and right posterior cingulate that was positively correlated with symptom severity.

These DTI findings are in line with the aforementioned studies in PD showing involvement of the left anterior and right posterior cingulate areas. The authors interpreted the increased white matter connectivity as being related to the increase in attention to specific external and internal stimuli in PD.

\section{Generalized Anxiety Disorder}

Generalized anxiety disorder (GAD) is characterized by excessive worrying and diffuse anxiety that are difficult to control. Patients with GAD may present with restlessness, fatigue, irritability, muscle tension, and sleep and concentration difficulties [1].

Neuroimaging data on GAD are relatively limited and sometimes unequivocal. The most consistent findings of brain abnormalities in GAD are probably hyperactivation of the amygdala and the medial prefrontal cortex [2].

We identified two recent studies that used DTI to examine brain abnormalities in GAD, both of them performed in a sample of adult patients. Hettema et al. [7] conducted a twin study with 17 pairs of females discordant for lifetime GAD. Several of the affected twins had also comorbid mood or anxiety disorders. The affected twins showed reduced FA values in the left uncinate fasciculus (UF) and in the right inferior longitudinal fasciculus. The UF connects the amygdala and the orbitofrontal cortex (OFC) [8]. The FA abnormalities in this region are consistent with studies in GAD in which abnormalities in connectivity between limbic and frontal structures (although not specifically involving the OFC) have been described $[9,10]$. Zhang et al. [11•] compared FA values in 20 treatment-naive patients with GAD without comorbidity and 28 healthy controls. They found higher FA values in the right postcentral gyrus of GAD patients. The postcentral gyrus is the location of the primary somatosensory cortex and was not implicated in GAD before, but these findings warrant further investigation.

\section{Social Anxiety Disorder}

Social anxiety disorder (SAD) is characterized by a marked and persistent fear of social or performance situations involving possible scrutiny by others [1]. Previous functional neuroimaging studies in patients with SAD have shown involvement of the amygdala, insula, and medial prefrontal regions. In SAD, the amygdala typically exhibits an exaggerated response in several social paradigms, including public speaking tasks and exposure to different facial expressions. 
Increased rostral ACC, orbital frontal, and insular activation have also been reported in several functional studies, but there are also contradictory findings [2]. Finally, dorsal ACC activation was found to be increased when confronted with negative comments [12] and harsh or disgusted facial expressions [13], and decreased when confronted with schematic angry faces [14] or in anticipation of public speaking. Volumetric MRI studies in SAD are very scarce and have yielded conflicting findings.

To date, three studies have utilized DTI to examine structural connectivity in patients with SAD. Liao et al. [15] examined 18 adult comorbidity and medication-free patients with SAD and matched healthy controls using a seed-based tractography. A significant increase in the FA values was observed within the genu of the corpus callosum (CC) when the seed ROI was located in the right medial prefrontal cortex. The authors interpreted this as an increased connectivity in cognitive reappraisal regions associated with long-term overgeneralization in SAD.

Phan et al. [16] examined 30 medication-free patients with SAD and 30 matched controls and found reduced FA values in the UF of SAD patients. This finding was partially replicated and extended by Baur et al. [17] in a study with 25 controls and 25 patients. In addition, the latter study found decreased connectivity in the left superior longitudinal fasciculus. The UF is a structural connection between the OFC and the amygdala. Reduced connectivity in the UF can be interpreted as reflecting or even underlying a diminished top-down control, leading to amygdala hyperactivation in SAD. Interestingly, studies in healthy individuals have shown a negative correlation between the FA values in the UF and measures for trait anxiety [18].

\section{Post-traumatic Stress Disorder}

Individuals with post-traumatic stress disorder (PTSD) re-experience a traumatic event in the form of nightmares, intrusive recollections, and flashbacks. Physiologic arousal and distress are displayed in response to reminders of trauma, which tend to be avoided. They also suffer from hyperarousal, difficulty sleeping, hypervigilance, and often a restricted range of affect [1].

A considerable number of functional and structural studies on PTSD have been conducted. There is consensus about the amygdala, medial prefrontal cortex (including the rostral and ventral ACC), and the hippocampus being key regions in the functional neurocircuitry implicated in PTSD. The available data support a model of the amygdala being hyperresponsive to trauma-related or fearful stimuli, with hyporesponsive medial prefrontal cortex areas failing to inhibit the amygdala. In addition, disturbed hippocampal function is supposed to lead to impairments in memory, contextual processing, and stress-axis dysregulation [2].
The available volumetric studies are inconclusive on volume reductions of the amygdala, but diminished volumes of the ACC and hippocampal regions are a more consistent finding in PTSD [2].

To date, five DTI studies in PTSD have been published. They have all included adult patients, and four of them included participants with comorbidity (mostly mood and anxiety disorders). The pattern of findings is somewhat mixed. Several studies have identified abnormalities in regions in the medial prefrontal cortex.

Two smaller studies have found decreased FA in the ACC of PTSD patients $[19,20]$. Additionally, Zhang et al. [11•] compared a sample of PTSD patients, GAD patients, and healthy controls with one another. It was demonstrated that a decreased FA in the ACC was more specific for PTSD patients. As an extension of their previous study, Kim et al. [21] examined ROIs in the cingulum bundle and found decreased FA in the left anterior cingulum bundle, whereas another very small study showed increased FA in the same region [22].

In the frontal lobe, decreased FA was found in the precentral gyrus [19]. White matter integrity in the left frontal gyrus was reported to be decreased [20] or increased [11•]. Finally, decreased FA was also observed in the internal capsule [19] and in the midbrain [20].

Interestingly, one study examined white matter connectivity in children with PTSD. Jackowski et al. [23] studied a sample of 17 maltreated, medication-free children with PTSD and 15 healthy controls and found a decreased FA in the body of the CC.

Abnormalities in structural integrity of cingulate regions, the cingulum bundle, and/or the amygdala are consistent with the existing literature on PTSD and may underlie or be the result of a disturbed "top-down" control. Findings in other regions of the frontal lobe are also partially in line with previous studies and interpreted as being related to disturbances in the recall and expression of extinction memory, a salient phenomenon in PTSD, or to disturbed emotion processing $[11 \cdot, 19]$.

\section{Obsessive-Compulsive Disorder}

Patients with obsessive-compulsive disorder (OCD) experience recurrent, unwanted thoughts or images (obsessions) that are egodystonic and cause distress. They engage in excessive ritualistic behaviors or mental acts (compulsions), typically carried out in response to the obsessions [1].

OCD had been studied longer and more extensively with neuroimaging techniques than most other anxiety disorders, and as a result, more elaborated models for the implicated neurocircuitry exist. The thalamo-cortico-striatal circuit (TCS) is thought to be central in the pathophysiology of OCD. The TCS comprises two interconnected pathways: the 
frontal-basal with the OFC and ACC, and the ganglia thalamic with the striatum and medial thalamic areas. Some elements of the previously discussed "fear circuitry," especially the amygdala, seem to be less implicated in neuroimaging studies of OCD [2].

A recent meta-analysis of structural volumetric MRI datasets found increased regional gray matter in the basal ganglia, probably increasing with more severe OCD, and decreased gray matter volumes in the frontal/anterior cingulate gyri [24]. Other studies have reported reduced volumes of the OFC and increased thalamic volumes.

Data from functional neuroimaging studies consistently support the involvement of the different elements of the TCS in OCD. Hyperactivity at rest and during symptom provocation was found in the cingulate cortex, and several studies showed dysfunctions of the OFC and abnormal activation in the basal ganglia [25]. Several abnormalities were reported to normalize with treatment.

To date, 11 studies exploring white matter connectivity with DTI in OCD have been published. The vast majority of studies included patients on medication, and in several studies, OCD patients differed in symptomatology or had comorbid disorders. Eight studies employed a whole brain approach. Three studies focused only on a specific location or specific tracts in the brain by employing ROI approaches. These examined the anterior segment of the cingulum bundle (CB), anterior thalamic radiations [26], the anterior limb of the internal capsule [27], and the CC [28].

Increased FA was found in right posterior [29] and medial [30] areas of the frontal lobe. Both findings in the medial cortex did not comprise the ACC. FA in the inferior parietal cortex was decreased unilaterally [30] or bilaterally [31]. The latter study also revealed decreased FA in the left occipital lobe.

Of 11 studies, 5 found abnormalities in the ACC and the $\mathrm{CB}$ [27, 31-34], while six did not report significant changes in this region [26, 29, 30, 35-37]. Data are especially conflicting for the left $\mathrm{ACC}$ and $\mathrm{CB}$, with some studies reporting increased FA [27, 34], others decreased FA [26, 31, 33], and several no change [29, 30, 32, 35-37]. One study reported decreased FA in the right posterior cingulate [31].

Of the 11 studies, seven reported abnormalities of the structural integrity of the $\mathrm{CC}$, but the data on direction of the changes and the specific location in the $\mathrm{CC}$ are conflicting. Two studies found decreased FA in the rostrum of the CC [28, 32] and one study found increased FA [37]. In the genu of the CC, studies found increased [29, 34] or decreased FA [32]. In the body of the CC, two studies revealed decreased [32, 35] and one increased FA [29]. Finally, in the splenium, one study showed increased [34] and another decreased FA [33].

Some studies reported changes in the structural integrity of areas other than the ACC, CB, or CC. Nakamae et al. [32] found increased FA in the bilateral semioval center extending to the subinsular white matter. Cannistraro et al. [27] found increased FA in the anterior limb of the internal capsule, while Nakamae et al. [32] reported a trend for lower FA in the same region. Interestingly, Yoo et al. [37] were the only ones to report abnormalities (an increase in FA) in the right caudate nucleus, a region often implicated previously in neuroimaging studies of OCD.

Finally, in the only DTI study in pediatric OCD, increased FA in several white matter tracts, including the major and minor forceps, left interior longitudinal fasciculus, bilateral corticospinal tract, uncinate fasciculus, right anterior thalamic radiations, and bilateral superior longitudinal fasciculus, was found [34]. However, Chiu et al. [26] reported decreased FA in the right anterior thalamic radiation, and Garibotto et al. [33] found decreased FA in the superior longitudinal fasciculus in adult OCD. The latter study also found decreased FA in the fronto-occipital fasciculus. Interestingly, in the only pre-/post-treatment study in OCD, Yoo et al. [37] investigated DTI abnormalities before and after treatment with citalopram and found that most abnormalities tended to diminish or disappear with treatment.

Taken together, DTI studies in OCD seem to implicate especially the ACC, $\mathrm{CB}$, and the $\mathrm{CC}$ in the pathophysiology of the disorder, although changes in these regions are not found consistently and often vary in direction. Some data also exist for abnormalities in the right posterior frontal lobe, uncinate fasciculus, inferior fronto-occipital fasciculus, anterior limb of internal capsule, anterior insula, caudate nucleus, and in anterior thalamic radiations. These tracts are relevant because of their involvement in or their connections to specific regions of the TCS.

\section{Internalizing Disorders in Children and Adolescents}

In children and adolescents, anxiety and depressive symptomatology are frequently comorbid and often referred to as the broader category of internalizing disorders, which also show neurobiological overlap. In a recent DTI study of our group examining white matter integrity in anxious and/or depressed adolescents, Aghajani et al. (unpublished data) employed voxel-wise whole brain analysis to search for group differences in FA. As part of the Emotional Pathways in Clinical Adolescents (EPISCA) project, DTI was performed in 25 treatment-naïve adolescents with internalizing disorders and 25 age- and gender-matched controls. Preliminary results indicate decreased FA values in the body of the $\mathrm{CC}$ in adolescents with internalizing disorders. Moreover, mean FA values within regions of the $\mathrm{CC}$ that showed significant group differences were negatively correlated with clinical measures of depression and anxiety in all participants. 


\section{Trait Anxiety}

Relevant to the understanding of the neurobiological underpinnings of anxiety disorders are findings in individuals with traits predisposing for the development of anxiety disorders. To date, there are three studies linking measures of trait anxiety or harm avoidance to DTI measures in healthy controls. Kim and Whalen [18] used the StateTrait Anxiety Inventory (STAI) and found that FA values of an amygdala-ventromedial prefrontal cortex pathway were negatively correlated with trait anxiety scores. The authors identified the UF as a putative candidate for this pathway. Westlye et al. [38••] used the Tridimensional Personality Questionnaire to specifically examine correlates of harm avoidance (HA), an anxiety trait associated with affective disorder. They examined a very large sample of healthy controls and found a negative association between HA and FA for $42 \%$ of all skeleton voxels, including critical hubs in the cortico-limbic neurocircuitry. Tractwise analysis highlighted the association of higher HA with lower FA in several important connections among others in the $\mathrm{CC}, \mathrm{CB}$, the corticospinal tract, forceps major and minor, inferior fronto-occipital fasciculus, inferior and superior longitudinal fasciculus, and UF. Baur et al. [17] used the trait version of the STAI in a group of both SAD patients and healthy controls and investigated the association with DTI measurement. They also found a negative correlation between FA and trait anxiety in the UF, but only in the patients and not in healthy controls. Thus far, these three studies on trait anxiety seem to point to a role for the UF, a major pathway between the amygdala and orbitofrontal lobe, in the neurobiological underpinnings of trait anxiety.

\section{Conclusions}

Thus far, the use of DTI has been very limited in PD, SAD, and GAD, and somewhat more extensive in PTSD and OCD. In most anxiety disorders, results of DTI studies are in line with other structural and functional MRI findings and can be interpreted in the context of existing models for the neurocircuitry of the various disorders. DTI findings could further enrich neurobiological models, although replication is often warranted. The most robust findings so far seem to be abnormalities of the structural integrity of the prefrontal cortex in PTSD and the ACC, CC, and CB in OCD. The very limited number of studies on trait anxiety seems to point to abnormalities in the UF, but these are not consistently reported in studies in anxiety disorders.

Surprisingly, until now, only one published study has investigated the effect of treatment on structural integrity as measured with DTI in anxiety disorders. DTI studies in pediatric populations and at-risk populations, together with DTI work on genetic influences in anxiety are currently also lagging behind, but rapid progress in these areas is expected, as acquisition of DTI is becoming more easy and more often incorporated into standard MRI research protocols.

Acknowledgments This review was supported through a EU Marie Curie International Staff Exchange Scheme grant for the European South African Research Network in Anxiety Disorders (EUSARNAD) (PIRSES-GA-2010-269213), and the Netherlands Organization for Scientific Research-National Initiative Brain and Cognition (NIBC) project grants no. 056-25-010 and no. 056-23-011.

Disclosure No potential conflicts of interest relevant to this article were reported.

\section{References}

Papers of particular interest, published recently, have been highlighted as:

- Of importance

-• Of major importance

1. American Psychiatric Association. Diagnostic and Statistical Manual of Mental Disorders: DSM-IV. 2000;Fourth Edition.

2. Shin LM, Liberzon I. The neurocircuitry of fear, stress, and anxiety disorders. Neuropsychopharmacology. 2010;35:169-91.

3. Asami T, Hayano F, Nakamura M, et al. Anterior cingulate cortex volume reduction in patients with panic disorder. Psychiatry Clin Neurosci. 2008;62:322-30.

4. Uchida RR, Del-Ben CM, Busatto GF, et al. Regional gray matter abnormalities in panic disorder: a voxel-based morphometry study. Psychiatry Res. 2008;163:21-9.

5. van Tol MJ, van der Wee NJ, van den Heuvel OA, et al. Regional brain volume in depression and anxiety disorders. Arch Gen Psychiatry. 2010;67:1002-11.

6. Han DH, Renshaw PF, Dager SR, et al. Altered cingulate white matter connectivity in panic disorder patients. J Psychiatr Res. 2008;42:399-407.

7. Hettema JM, Kettenmann B, Ahluwalia V et al. Pilot multimodal twin imaging study of generalized anxiety disorder. Depress Anxiety. 2011.

8. Ebeling U, von CD. Topography of the uncinate fascicle and adjacent temporal fiber tracts. Acta Neurochir (Wien). 1992;115:143-8.

9. Etkin A, Prater KE, Schatzberg AF, et al. Disrupted amygdalar subregion functional connectivity and evidence of a compensatory network in generalized anxiety disorder. Arch Gen Psychiatry. 2009;66:1361-72.

10. Etkin A, Prater KE, Hoeft F, et al. Failure of anterior cingulate activation and connectivity with the amygdala during implicit regulation of emotional processing in generalized anxiety disorder. Am J Psychiatry. 2010;167:545-54.

11. - Zhang L, Zhang Y, Li L et al. Different white matter abnormalities between the first-episode, treatment-naive patients with posttraumatic stress disorder and generalized anxiety disorder without comorbid conditions. J Affect Disord. 2011;133:294-299. This study also examined differences between anxiety disorders.

12. Blair K, Geraci M, DeVido J, et al. Neural response to self- and other referential praise and criticism in generalized social phobia. Arch Gen Psychiatry. 2008;65:1176-84.

13. Amir N, Klumpp H, Elias J, et al. Increased activation of the anterior cingulate cortex during processing of disgust faces in individuals with social phobia. Biol Psychiatry. 2005;57:975-81. 
14. Evans KC, Wright CI, Wedig MM, et al. A functional MRI study of amygdala responses to angry schematic faces in social anxiety disorder. Depress Anxiety. 2008;25:496-505.

15. Liao W, Xu Q, Mantini D, et al. Altered gray matter morphometry and resting-state functional and structural connectivity in social anxiety disorder. Brain Res. 2011;1388:167-77.

16. Phan KL, Orlichenko A, Boyd E, et al. Preliminary evidence of white matter abnormality in the uncinate fasciculus in generalized social anxiety disorder. Biol Psychiatry. 2009;66:691-4.

17. Baur V, Hanggi J, Rufer M, et al. White matter alterations in social anxiety disorder. J Psychiatr Res. 2011;45:1366-72.

18. Kim MJ, Whalen PJ. The structural integrity of an amygdala-prefrontal pathway predicts trait anxiety. J Neurosci. 2009;29:11614-8.

19. Schuff N, Zhang Y, Zhan W, et al. Patterns of altered cortical perfusion and diminished subcortical integrity in posttraumatic stress disorder: an MRI study. Neuroimage. 2011;54 Suppl 1:S62-8.

20. Kim MJ, Lyoo IK, Kim SJ, et al. Disrupted white matter tract integrity of anterior cingulate in trauma survivors. Neuroreport. 2005;16:1049-53.

21. Kim SJ, Jeong DU, Sim ME, et al. Asymmetrically altered integrity of cingulum bundle in posttraumatic stress disorder. Neuropsychobiology. 2006;54:120-5.

22. Abe O, Yamasue H, Kasai K, et al. Voxel-based diffusion tensor analysis reveals aberrant anterior cingulum integrity in posttraumatic stress disorder due to terrorism. Psychiatry Res. 2006;146:231-42.

23. Jackowski AP, Douglas-Palumberi H, Jackowski M, et al. Corpus callosum in maltreated children with posttraumatic stress disorder: a diffusion tensor imaging study. Psychiatry Res. 2008;162:256-61.

24. Radua J, Mataix-Cols D. Voxel-wise meta-analysis of grey matter changes in obsessive-compulsive disorder. Br J Psychiatry. 2009;195:393-402.

25. Del CA, Kotzalidis GD, Rapinesi C, et al. Functional neuroimaging in obsessive-compulsive disorder. Neuropsychobiology. 2011;64:61-85.

26. Chiu CH, Lo YC, Tang HS, et al. White matter abnormalities of fronto-striato-thalamic circuitry in obsessive-compulsive disorder: a study using diffusion spectrum imaging tractography. Psychiatry Res. 2011;192:176-82.

27. Cannistraro PA, Makris N, Howard JD, et al. A diffusion tensor imaging study of white matter in obsessive-compulsive disorder. Depress Anxiety. 2007;24:440-6.
28. Saito Y, Nobuhara K, Okugawa G, et al. Corpus callosum in patients with obsessive-compulsive disorder: diffusion-tensor imaging study. Radiology. 2008;246:536-42.

29. Li F, Huang X, Yang Y, et al. Microstructural brain abnormalities in patients with obsessive-compulsive disorder: diffusiontensor MR imaging study at 3.0 T. Radiology. 2011;260:21623.

30. Menzies L, Williams GB, Chamberlain SR, et al. White matter abnormalities in patients with obsessive-compulsive disorder and their first-degree relatives. Am J Psychiatry. 2008;165:130815.

31. Szeszko PR, Ardekani BA, Ashtari M, et al. White matter abnormalities in obsessive-compulsive disorder: a diffusion tensor imaging study. Arch Gen Psychiatry. 2005;62:782-90.

32. Nakamae T, Narumoto J, Sakai Y, et al. Diffusion tensor imaging and tract-based spatial statistics in obsessive-compulsive disorder. J Psychiatr Res. 2011;45:687-90.

33. Garibotto V, Scifo P, Gorini A, et al. Disorganization of anatomical connectivity in obsessive compulsive disorder: a multi-parameter diffusion tensor imaging study in a subpopulation of patients. Neurobiol Dis. 2010;37:468-76.

34. Zarei M, Mataix-Cols D, Heyman I, et al. Changes in gray matter volume and white matter microstructure in adolescents with obsessive-compulsive disorder. Biol Psychiatry. 2011;70:1083-90.

35. Bora E, Harrison BJ, Fornito A, et al. White matter microstructure in patients with obsessive-compulsive disorder. J Psychiatry Neurosci. 2011;36:42-6.

36. Nakamae T, Narumoto J, Shibata K, et al. Alteration of fractional anisotropy and apparent diffusion coefficient in obsessive-compulsive disorder: a diffusion tensor imaging study. Prog Neuropsychopharmacol Biol Psychiatry. 2008;32:1221-6.

37. Yoo SY, Jang JH, Shin YW, et al. White matter abnormalities in drug-naive patients with obsessive-compulsive disorder: a diffusion tensor study before and after citalopram treatment. Acta Psychiatr Scand. 2007;116:211-9.

38. •- Westlye LT, Bjornebekk A, Grydeland H et al. Linking an anxiety-related personality trait to brain white matter microstructure: diffusion tensor imaging and harm avoidance. Arch Gen Psychiatry. 2011;68:369-377. This study was conducted in a large sample of healthy individuals. 